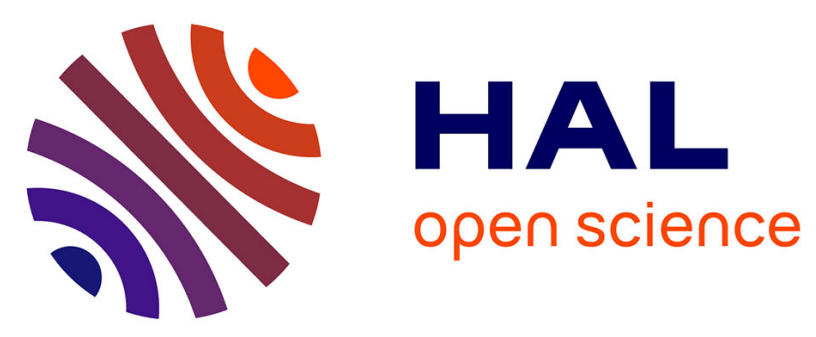

\title{
Relations entre le nombre de mâles de l'eudémis de la vigne, Lobesia botrana Den. \& Schiff. (Lepid., Tortricidae), capturés par deux doses de phéromone et le niveau des populations larvaires dans les parcelles de piégeage
}

Roger Roehrich, Jean-Paul Carles, Hélène Durand, Jean-Luc Tymen

\section{To cite this version:}

Roger Roehrich, Jean-Paul Carles, Hélène Durand, Jean-Luc Tymen. Relations entre le nombre de mâles de l'eudémis de la vigne, Lobesia botrana Den. \& Schiff. (Lepid., Tortricidae), capturés par deux doses de phéromone et le niveau des populations larvaires dans les parcelles de piégeage. Agronomie, 1986, 6 (5), pp.447-452. hal-00884895

\section{HAL Id: hal-00884895 \\ https://hal.science/hal-00884895}

Submitted on 1 Jan 1986

HAL is a multi-disciplinary open access archive for the deposit and dissemination of scientific research documents, whether they are published or not. The documents may come from teaching and research institutions in France or abroad, or from public or private research centers.
L'archive ouverte pluridisciplinaire HAL, est destinée au dépôt et à la diffusion de documents scientifiques de niveau recherche, publiés ou non, émanant des établissements d'enseignement et de recherche français ou étrangers, des laboratoires publics ou privés. 


\section{Relations entre le nombre de mâles de l'eudé- mis de la vigne, Lobesia botrana Den. \& Schiff. (Lepid., Tortricidae), capturés par deux doses de phéromone et le niveau des populations lar- vaires dans les parcelles de piégeage}

Roger ROEHRICH, Jean-Paul CARLES, Hélène DURAND \& Jean-Luc TYMEN

I.N.R.A., Station de Zoologie, Centre de Recherches de Bordeaux, F 33140 Pont-de-la-Maye

RÉSUMÉ Dans plusieurs parcelles d'un vignoble, des pièges sexuels sont appâtés alternativement au moyen de 2 doses
différentes de phéromone.
En comparant le nombre de papillons capturés au pourcentage d'infestation par les chenilles de la génération
suivante, on obtient pour la $2^{\mathrm{e}}$ génération, une assez bonne corrélation pour les doses les plus faibles : ceci
implique que les papillons capturés proviennent, dans ce cas, en plus grande proportion de l'environnement
proche. Des doses faibles seraient donc à préférer pour une approche quantitative et, en particulier, pour amé-
liorer la prévision négative actuellement utilisée pour déterminer les parcelles où une intervention est inutile.

Mots clés additionnels : Prognose, avertissements agricoles. dosages and the larval population in the trapping plots.

Sexual traps were set out, with two alternating pheromone doses, in several plots of a vineyard. A significant correlation was found between the number of males trapped at the lower dosage and the number of larvae per 100 bunches. This implied that most of the moths trapped came from the immediate environment. Low dosage would thus be preferable for quantitative assessments and especially to improve the negative forecasting currently used to decide that treatment is not needed in a plot.

Additional key words : Prognosis, forecasting.

\section{INTRODUCTION}

Depuis que de nombreuses phéromones de lépidoptères nuisibles sont disponibles, la possibilité de les utiliser pour évaluer les risques d'intervention a été continuellement recherchée. Dans certains cas, cela a été possible. AUDEMARD (1979) a établi une méthode permettant d'aménager la lutte chimique contre le carpocapse (Laspeyresia pomonella L.) grâce à la comparaison des captures pendant une semaine avec des seuils d'intensification ou de diminution établis selon les conditions des régions; mais un tel résultat est assez rare en culture intensive. En forêt, nous pouvons citer, par exemple, la bonne corrélation entre le piégeage au moyen de pièges non saturables et l'infestation de tordeuse de l'épinette, Choristoneura fumiferana Clem., obtenue par RAMASWANY \& CARDÉ (1983). Mais pour les tordeuses de la grappe et, en particulier, pour l'eudémis (Lobesia botrana Den. \& Schiff.), les expériences ont montré de grandes disparités dans les résultats et seule une prévision négative s'est montrée possible dans certains cas (ROEHRICH \& SCHMID, 1979). L'hypothèse d'une meilleure concordance entre les piégeages et l'infestation consécutive lorsqu'on utilise des diffuseurs renfermant de plus faibles quantités de phéromones a été avancée par VALLI en 1977 et des expérimentations ont été effectuées en France avec des « minidoses » de phéromones sur dif- 
férents insectes par le Service de la Protection des Végétaux (BESSON, 1981). Nous avons utilisé une autre approche, en piégeant dans les mêmes parcelles, alternativement, au moyen de 2 doses de phéromones pour rechercher laquelle des deux donne une meilleure image de l'infestation par la descendance.

\section{MATÉRIEL ET MÉTHODES}

Le composé phéromonal (acétoxy-1-dodécadiène 7E, 9Z), le type de piège (piège I.N.R.A.), les diffuseurs (bouchons de caoutchouc), la périodicité de leur renouvellement (à chaque génération) sont les mêmes que dans les essais ayant fait l'objet d'une précédente note (ROEHRICH et al., 1983).

L'expérimentation est implantée au Château Baret, vignoble de la région «Graves » situé à Villenave-d'Ornon. La figure 1 donne le plan du vignoble et l'encépagement des différentes parcelles; selon les années et les vols, nous en avons utilisé de 4 à 10. Au centre de chacune, un piège sexuel est appâté alternativement avec chacune des 2 doses de phéromone. Pour que les résultats puissent être comparés à ceux d'un piégeage continu, le nombre de mâles piégés est multiplié par 2 si la dose est présentée 1 jour sur 2 , par 4 si elle est présentée 1 jour sur 4 .

\begin{tabular}{|c|c|c|c|c|c|c|c|}
\hline $\mathrm{CAB}$ & & MER & & SAU & & & $N$ \\
\hline MER & $c 4$ & MER & $\mathrm{C}_{3}$ & SEM & $c 2$ & SAU & \\
\hline 1981 & 1982 & & 1982 & 1981 & 1982 & & \\
\hline 1 & 1 & & - & - & 1 & & \\
\hline 2 & 2 & & $\begin{array}{l}2 \\
3\end{array}$ & $\begin{array}{l}2 \\
3\end{array}$ & 2 & & \\
\hline SEM & $B 4$ & $C A B+M A L$ & 83 & SAU & B 2 & SEM & B1 \\
\hline 1981 & 1982 & 1981 & 1982 & 1981 & 1982 & 1981 & \\
\hline- & - & 1 & 1 & - & 1 & - & \\
\hline 2 & 2 & 2 & 2 & 2 & 2 & 2 & \\
\hline \multirow[t]{6}{*}{3} & 3 & 3 & 3 & 3 & 3 & 3 & \\
\hline & DIVERS & $C A B$ & A 3 & $\underline{\operatorname{SAU}}$ & A 2 & $\mathrm{CAB}+\mathrm{SEM}$ & A1 \\
\hline & $1981^{A 4}$ & 1981 & 1982 & $\overline{1981}$ & 1982 & 1981 & \\
\hline & 1 & - & - & - & - & 1 & \\
\hline & - & 2 & 2 & 2 & 2 & - & \\
\hline & _- & 3 & 3 & 3 & 3 & - & \\
\hline
\end{tabular}

Figure 1

Implantation des essais dans les parcelles de vignoble. Cépages (les blancs sont soulignés) : $C A B .=$ Cabernet ; $M A L .=$ Malbec ; $M E R .=$ Merlot $;$ SAU. = Sauvignon $;$ SEM. = Sémillon.

Location of the experiments in the plots of the vineyard. Grape cultivars (the white ones are underlined).

Les doses choisies ont été, en 1981, $1000 \mu \mathrm{g}$, dose utilisée par les avertissements agricoles et $10 \mu \mathrm{g}$, et, en $1982,10 \mu \mathrm{g}$ et $1 \mu \mathrm{g}$.

L'infestation est évaluée à la fin des $1^{\text {er }}$ et $2^{\text {e }}$ vols par contrôle visuel sur les grappes. En $1^{\text {re }}$ génération, on note le nombre des glomérules, en $2^{\mathrm{e}}$ génération le nombre de points d'attaque (groupes de grains endommagés). L'échantillon est composé de 1 à 5 ceps consécutifs sur lesquels on observe, lors de la $1^{\text {re }}$ génération, toutes les grappes et ensuite 4 grappes par cep, la croissance de la végétation rendant difficile l'accès à toutes les grappes. Le contrôle a été effectué sur 300 à 1500 grappes par parcelle selon l'infestation. En $3^{\text {e }}$ génération, le contrôle visuel des attaques n'est plus possible. Nous avons dénombré les œufs visibles sur les grappes à la suite de 3 passages espacés d'une semaine.

\section{RÉSULTATS}

Le tableau 1 donne le nombre de mâles capturés au moyen de chacune des doses, dans chaque parcelle, pour les 3 vols des 2 années 1981 et 1982. On peut remarquer que, sauf lors du $1^{\text {er }}$ vol de 1981, malgré une disparité entre les sites de piégeage, les 2 doses ont capturé, pour l'ensemble du vignoble, des nombres très voisins de mâles.

TABLEAU 1

Piégeages des mâles d'eudémis au cours des 3 vols en 1981 et 1982. Trapping of males during the 3 flight periods in 1981 and 1982.

\begin{tabular}{|c|c|c|c|c|}
\hline \multicolumn{2}{|c|}{$\begin{array}{l}\text { Doses comparées } \\
\text { (concentrations) }\end{array}$} & $\frac{1000 \mu \mathrm{g}}{518}$ & $\frac{10 \mu \mathrm{g}}{715}$ & \multirow[t]{2}{*}{$1 \mu \mathrm{g}$} \\
\hline 1981 & $\begin{array}{l}1^{\text {er }} \text { vol } \\
2^{e} \text { vol } \\
3^{\text {e }} \text { vol }\end{array}$ & $\begin{array}{l}518 \\
139 \\
112\end{array}$ & $\begin{array}{l}715 \\
169 \\
108\end{array}$ & \\
\hline 1982 & $\begin{array}{l}1^{\mathrm{er}} \mathrm{vol} \\
2^{\mathrm{e}} \mathrm{vol} \\
3^{\mathrm{e}} \mathrm{vol}\end{array}$ & & $\begin{array}{l}160 \\
179 \\
426\end{array}$ & $\begin{array}{r}54 \\
219 \\
422\end{array}$ \\
\hline
\end{tabular}

La comparaison de ces captures avec les effectifs de chenilles peut être envisagée de 2 façons: avec la génération précédente d'où proviennent les papillons piégés ou avec la génération suivante.

Il est possible de comparer les captures des $2^{e}$ et $3^{\mathrm{e}}$ vols à un indice évaluant la population mâle dans les parcelles où sont effectuées ces captures (tabl. 2). Cet indice, égal à la moitié du nombre des attaques constatées lors de la génération précédente, est cependant surévalué par rapport à la population réelle puisqu'il ne tient pas compte de la mortalité nymphale. Toute évaluation est impossible pour le $1^{\text {er }}$ vol (qui correspond à la génération hivernante) : en effet, il n'y a pas de relation entre le nombre de chenilles présentes à l'automne et le nombre de papillons présents au printemps.

Au $2^{\mathrm{e}}$ vol, il apparaît nettement que le pourcentage des captures est plus élevé lorsque la parcelle a été peu infestée lors de la génération précédente (C 4 en 1981 et B 3 en 1984) : si les captures révèlent bien la distribution des papillons, ceux-ci sont donc répartis d'une façon plus homogène que les chenilles de $1^{\text {re }}$ génération. Au cours du $3^{\mathrm{e}}$ vol, il y a encore des disparités importantes dans les pourcentages (de 2,4 à 17,2 p. 100 en 1981 ; de 2,2 à 13,1 p. 100 en 1982), mais il n'y a pas de relation simple avec la population de chenilles.

Les piégeages pourraient donc nous renseigner non sur la position des papillons au moment de l'émergence, mais sur leur position au moment de la ponte. Il est donc important de comparer le nombre de papillons capturés au nombre de chenilles de la génération suivante ou à un autre indice variant en même temps 
TABLEAU 2

Effectifs de mâles capturés par piège lors des $2^{e}$ et $3^{e}$ vols exprimés en pourcentage du nombre maximal des mâles provenant de 1 ha.

Number of males caught per trap during the 2nd and 3 rd flights $1 \%$ of the maximum number of males per 1 ha).

(1) Evaluation du nombre maximal de mâles par ha : attaques lors de la génération précédente divisées par 2.

Evaluation of the maximum number of males in one ha (infestation by the preceding generation, divided by 2).

(2), (3), (4) Mâles capturés p. 100 du nombre maximal évalué dans les pièges appâtés avec $1000 \mu \mathrm{g}(2), 10 \mu \mathrm{g}$ (3) et $1 \mu \mathrm{g}$ (4) de phéromone. Trapped males \% of the maximal evaluated number in traps of I $000 \mu g(2), 10 \mu g(3), I \mu g$ (4) of pheromone.

\begin{tabular}{|c|c|c|c|c|c|c|}
\hline \multirow{2}{*}{$\begin{array}{l}\text { Parcelles } \\
\text { Plots }\end{array}$} & \multicolumn{3}{|c|}{1981} & \multicolumn{3}{|c|}{1982} \\
\hline & \multicolumn{6}{|c|}{$2^{e} \mathrm{vol}$} \\
\hline & (1) & (2) & (3) & (1) & (3) & (4) \\
\hline A 1 & 1750 & 2,4 & 4,7 & - & - & - \\
\hline A 2 & - & - & - & 850 & 3,8 & 1,9 \\
\hline A 3 & 1100 & 3,6 & 2,4 & 1550 & 2,5 & 2,3 \\
\hline B 1 & 1100 & 8,5 & 3,5 & - & - & - \\
\hline B 2 & 2200 & 3,4 & 3,7 & 1000 & 3,4 & 2,4 \\
\hline B 3 & 1750 & 1,5 & 0,8 & 1800 & 3,1 & 1,6 \\
\hline B 4 & - & - & - & 250 & 12,0 & 18,4 \\
\hline C 2 & 1650 & 4,1 & 1,1 & 1750 & 4,5 & 3,0 \\
\hline C 3 & - & - & - & 2000 & 4,7 & 2,4 \\
\hline \multirow[t]{2}{*}{ C 4} & 500 & 30,0 & 20,0 & 1750 & 4,5 & 6,1 \\
\hline & \multicolumn{6}{|c|}{$3^{e}$ vol } \\
\hline A 2 & 1350 & 17,2 & 2,4 & 1150 & 12,1 & 7,3 \\
\hline A 3 & 1700 & 2,4 & 4,7 & 2500 & 7,2 & 10,2 \\
\hline B 1 & 1350 & 12,1 & 12,4 & - & - & - \\
\hline B 2 & 1100 & 3,3 & 10,8 & 1450 & 8,3 & 6,8 \\
\hline B 3 & 2100 & 7,6 & 3,0 & 2700 & 11,4 & 5,0 \\
\hline B 4 & 1250 & 3,8 & 9,6 & 2900 & 13,1 & 5,6 \\
\hline $\mathrm{C} 2$ & 2200 & 6,0 & 7,5 & 2850 & 6,2 & 5,8 \\
\hline C 3 & - & - & - & 8450 & 2,2 & 2,0 \\
\hline C 4 & 2950 & 2,3 & 3,9 & 4000 & 8,5 & 10,4 \\
\hline
\end{tabular}

que celui-ci (points d'attaque observés, nombre d'œufs).

En $1^{\text {re }}$ génération, les répétitions sont insuffisantes pour fournir des données exploitables. De plus, les infestations ont atteint sensiblement le même niveau dans 2 parcelles sur 4 en 1981 et 3 parcelles sur 4 en 1982, soit 3500 à 3600 glomérules par ha. Dans ces 5 parcelles, pour un même dosage en phéromone $(10 \mu \mathrm{g})$, un papillon capturé correspond à 10 glomérules en 1981 et à 44 en 1982 . Cette différence peut être expliquée par les conditions météorologiques : en 1981, des températures élevées pendant une courte période lors de la sortie des adultes, ont permis de nombreuses captures mais, de trop courte durée, elles n'ont pu permettre le dépôt des œufs sur des grappes réceptives.

$\mathrm{Au}$ cours des générations d'été au contraire, il est possible de comparer captures et niveau d'infestation dans chaque parcelle (tabl. 3).

En $2^{\mathrm{e}}$ génération, le quotient entre l'infestation et le nombre de mâles capturés est moins hétérogène lorsque les doses employées sont plus faibles et il en résulte une valeur plus faible du rapport de la racine carrée de la variance à la moyenne. La corrélation entre les captures par la dose faible et le pourcentage de grappes attaquées est significative en 1982 et le coefficient de corrélation $(0,85)$ indique que 72 p. 100 de la relation entre ces 2 variables est expliquée, mais aucune autre corrélation entre les captures et le nombre évalué de chenilles n'est significative.

En $3^{\mathrm{e}}$ génération au contraire, la variance des résul- tats est plus élevée et sensiblement la même quel que soit le dosage en phéromone de l'appât.

Quelle que soit la concentration de l'appât, aucune relation n'apparaît entre le nombre de mâles capturés et l'infestation : en effet, les indices des colonnes 3,4 et 5 sont, dans tous les cas, très variés. Il n'est donc pas possible d'utiliser le piégeage pour décider s'il faut ou non traiter la parcelle.

\section{DISCUSSION}

Après avoir discuté la valeur du dispositif expérimental utilisé, nous examinerons dans quelle mesure les résultats obtenus permettent de progresser vers le but : l'amélioration de la prévision quantitative.

\section{A. Critiques du dispositif expérimental}

L'éloignement des pièges doit limiter les risques de concurrence entre eux, mais le principe même de l'alternance pourrait apporter certaines erreurs systématiques.

Le risque de contamination, par la phéromone dégagée et déposée sur le feuillage, de la zone des pièges renfermant les diffuseurs les plus chargés, paraît très faible. Pour concurrencer ou renforcer l'appât, il faudrait qu'il s'évapore, à partir de ces sites de condensation, des quantités de phéromone non négligeables par rapport à celles provenant des diffuseurs les 
TABLEAU 3

Relation entre le nombre de mâles capturés et l'infestation par les chenilles de la génération suivante. Relation between number of trapped moths and larval infestation in the next generation.

(1), (2) Nombre de points de pénétration des chenilles ( $2^{e}$ génération) ou nombre d'œufs (3énération) pour lo0 grappes (1) et par lo0 ares (2). Number of points of entry (2nd generation) or number of eggs (3rd) in 100 bunches (1) and in I00 ares (2).

(3), (4), (5) Nombre de points de pénétration de chenilles ou nombre d'œufs correspondant à un mâle capturé par diffuseur chargé à 1 ug (3), $10 \mu \mathrm{g}$ (4) et $1000 \mu \mathrm{g}$ (5) de phéromones. Données ramenées à un piégeage continu pendant tout le vol. Number of points of entry or number of eggs corresponding to one male trapped per dispenser with $1 \mu \mathrm{g}(3), 10 \mu \mathrm{g}(4)$ and $1000 \mu \mathrm{g}(5)$ of pheromone. Data calculated for continuous trapping during the whole period of moth activity.

\begin{tabular}{|c|c|c|c|c|c|c|c|c|}
\hline \multirow{2}{*}{$\begin{array}{c}\text { Parcelles et cépages } \\
\text { Plots and varieties }\end{array}$} & \multicolumn{4}{|c|}{1981} & \multicolumn{4}{|c|}{1982} \\
\hline & (1) & (2) & (4) & (5) & (1) & (2) & (3) & (4) \\
\hline & \multicolumn{8}{|c|}{ 2è génération } \\
\hline A 1 Sém. Cab. $\left({ }^{*}\right)$ & 12 & 56 & 1,3 & 0,7 & - & - & - & - \\
\hline A 2 Sau. & 11 & 27 & 0,4 & 0,5 & 9 & 23 & 1,4 & 1,3 \\
\hline A 3 Cab. & 10 & 34 & 0,9 & 1,3 & 13 & 50 & 1,4 & 1,4 \\
\hline B 1 Sém. & 15 & 27 & 0,3 & 0,7 & - & - & - & - \\
\hline B 2 Sau. & 9 & 22 & 0,3 & 0,3 & 5 & 19 & 0,5 & 0,8 \\
\hline B 3 Cab. Mal. & 11 & 42 & 1,6 & 3,0 & 12 & 54 & 2,1 & 1,3 \\
\hline B 4 Sém. & 12 & 25 & 0,8 & 0,4 & 10 & 58 & 1,2 & 1,2 \\
\hline C 2 Sém. & 22 & 44 & 0,7 & 1,8 & 15 & 47 & 1,2 & 1,2 \\
\hline C 3 Mer. & - & - & - & - & 27 & 169 & 1,9 & 3,5 \\
\hline C 4 Mer. & 12 & 59 & 0,4 & 0,6 & 15 & 80 & 1,0 & 0,8 \\
\hline Moyenne $(\overline{\mathrm{m}})$ & 13 & 37 & 0,7 & 1,0 & 13 & 62 & 1,3 & 1,4 \\
\hline \multirow[t]{2}{*}{$\frac{\sqrt{v}}{m}$} & & & $\underline{0.59}$ & $\underline{0,81}$ & & & $\underline{0,37}$ & $\underline{0,52}$ \\
\hline & \multicolumn{8}{|c|}{$3^{\mathrm{e}}$ génération } \\
\hline A $2 \mathrm{Sau}$ & 142 & 349 & 1,5 & 14,5 & 272 & 695 & 19,3 & 24,8 \\
\hline A $3 \mathrm{Cab}$. & 17 & 56 & 1,4 & 0,7 & 159 & 612 & 15,3 & 10,9 \\
\hline B 1 Sém. & 180 & 322 & 2,0 & 1,9 & - & - & - & - \\
\hline B 2 Sau. & 172 & 475 & 13,2 & 3,0 & 161 & 612 & 17,5 & 20,4 \\
\hline B 3 Cab. Mal. & 27 & 101 & 0,6 & 1,6 & 228 & 1026 & 12,7 & 36,7 \\
\hline B 4 Sém. & 71 & 147 & 3,1 & 1,2 & 200 & 1160 & 34,1 & 15,9 \\
\hline C 2 Sém. & 195 & 385 & 2,9 & 2,3 & 288 & 902 & 22,0 & 22,6 \\
\hline C 3 Mer. & - & - & - & - & 142 & 888 & 16,4 & 17,1 \\
\hline C 4 Mer. & 29 & 139 & 2,0 & 1,3 & 159 & 848 & 10,5 & 9,8 \\
\hline Moyenne $(\overline{\mathrm{m}})$ & 104 & 229 & 3,3 & 3,3 & 201 & 843 & 18,5 & 19,8 \\
\hline$\frac{\sqrt{\mathrm{v}}}{\mathrm{m}}$ & & & 1,16 & 1,29 & & & $\underline{1,04}$ & $\underline{1,15}$ \\
\hline
\end{tabular}

(*) Sém. = Sémillon ; Cab. = Cabernet ; Sau. = Sauvignon ; Mal. = Malbec ; Mer. = Merlot.

moins chargés. Des essais de pesées concernant des doses plus élevées ont montré que le diffuseur libère quotidiennement environ 1 p. 100 de sa charge (ROEHRICH \& SCHMID, 1979). Le rapport des doses utilisées en 1981 étant de 1 à 100 , il aurait fallu que toute la phéromone diffusée pendant une journée se dépose et soit libérée le lendemain pour doubler la quantité présente dans l'air à ce moment. De plus, d'autres informations démontrent que ces doses sont capables d'attirer les papillons : d'abord la dose faible de 1981 correspond à la dose forte de 1982 et ensuite, les essais effectués par le Service de la Protection des Végétaux avec des diffuseurs chargés de $10 \mu \mathrm{g}$, sans autre témoin que des femelles, ont capturé des quantités d'insectes comparables ou supérieures à celles que l'on capture en général avec la dose commerciale de $1000 \mu \mathrm{g}$ (BESSON, 1981).

\section{B. Possibilités d'utilisation du piégeage dans la straté- gie de protection du vignoble}

Il se confirme, dans la limite des niveaux de populations effectivement observés lors de ces essais, qu'il n'y a pas de relation évidente entre le nombre de papillons capturés et le risque encouru par les cultu- res. En diminuant la dose, on diminue l'hétérogénéité du risque par individu capturé, mais les données obtenues ne permettent pas de prévoir le développement de la population, donc de déterminer la conduite à tenir dans la parcelle.

Avec Grapholitha molesta Busck, BAKER \& ROELOFS (1981) ont étudié le comportement de l'insecte lorsqu'il est sollicité par la phéromone et qu'il arrive aux alentours du piège. Ils ont pu conclure que des doses plus faibles que celles habituellement utilisées sont susceptibles de donner de meilleures informations sur l'activité locale des papillons puisqu'elles ne les attirent pas depuis de trop grandes distances.

Il apparaît que ce modèle ne peut pas s'appliquer au cas qui nous intéresse, dans un vignoble renfermant plusieurs parcelles différentes par la situation, l'encépagement et l'âge de la plantation : même en réduisant de 1000 fois la dose, il n'est pas possible de limiter la capture aux seuls insectes originaires de la parcelle piégée. Si le signal n'est pas perceptible audelà, les déplacements dus à l'errance dans le vignoble, à l'attirance de la végétation selon le stade de développement peuvent amener d'autres mâles dans le rayon d'action de l'appât le moins concentré. Une confirmation de ce fait peut être trouvée dans nos observations sur la capture après automarquage dans 
un piège sexuel restant appâté. On a pu recapturer 43 p. 100 des insectes ainsi marqués. Or 27 p. 100 de ces recaptures ont été effectuées entre 50 et $75 \mathrm{~m}$, soit à l'intérieur d'une surface de 0,8 à 1,8 ha autour du lieu de marquage et 7 p. 100 ont même été repris à plus de $300 \mathrm{~m}$, soit à l'intérieur d'une surface de 28 ha (ROEHRICH, 1981). Dans ce cas, le papillon étant à son envol à proximité immédiate de son piège de marquage, la première partie de son déplacement au moins est sous l'influence d'autres facteurs que la phéromone.

Des essais pour rapporter les données du piégeage au niveau de l'infestation consécutive ont souvent été effectués (ROEHRICH \& SCHMID, 1979), mais sans résultat généralisable. Dans une région, le classement des vignobles d'après le nombre de papillons capturés n'est pas le même que le classement d'après le nombre de chenilles infestantes. En particulier, il dépend de l'environnement et pour un même niveau d'infestation, on capture plus de mâles dans les vignobles faisant partie d'une région viticole que dans les vignobles isolés. BRESCHBUHLER (cf. ROEHRICH, 1981) a essayé de déterminer une relation mathématique et une courbe peut parfois être calculée avec une probabilité satisfaisante, mais, d'une part, les paramètres varient selon les années, d'autre part, la dispersion des résultats autour de la courbe ne permet pas de les employer pour conseiller, sans risque pour la récolte, le nontraitement de parcelles où il y a eu assez de captures. On a plutôt tenté d'établir des seuils d'intervention, en cherchant jusqu'à quelles limites on peut étendre la notion de prévision négative : s'il n'y a pas eu de captures, il n'y aura pas de dommages, les attaques restant au-dessous du seuil de nuisibilité (ROEHRICH \& SCHMID, 1979).

Pour la Suisse par exemple, ils ont montré que, audessous de 100 papillons capturés au cours du $1^{\mathrm{er}} \mathrm{vol}$, il y a moins de 15 p. 100 de grappes attaquées et qu'il n'est pas utile de traiter; s'il y a plus de 100 papillons, dans de nombreux cas le traitement est également inutile, mais on le sait trop tard. MAURIN (1980) a suggéré, pour le $2^{\mathrm{e}} \mathrm{vol}$ en Gironde, un raisonnement analogue en proposant provisoirement un seuil de 10 papillons pendant la $1^{\text {re }}$ semaine de piégeage. Que peut donc apporter l'utilisation de diffuseurs à faible dosage pour améliorer la prévision ? Il est impossible au niveau d'une région de classer les vignobles d'après le nombre de papillons capturés. Nous avions envisagé qu'il aurait peut-être été possible de classer les parcelles dans le cadre d'un vignoble ; l'essai n'a pas confirmé cette hypothèse, mais on a pu constater que les différences d'infestation entre parcelles ont été toujours relativement faibles, probablement trop pour permettre une bonne discrimination.

Néanmoins, on peut espérer améliorer l'utilisation de la prévision négative pour les générations se développant après nouaison et avant la véraison des raisins. En effet, nous avons montré que la dispersion des rapports $\frac{\text { dommages }}{\text { captures }}$ est nettement moindre lorsqu'on utilise des doses faibles. Elle est cependant loin d'être négligeable et il ne paraît pas que la notion de prévision négative puisse être remplacée par une évaluation de la population. L'usage des diffuseurs moins chargés $(10 \mu \mathrm{g}$ et surtout $1 \mu \mathrm{g})$ doit diminuer le nombre de cas où l'on capture un nombre de papillons supérieur au seuil d'intervention tandis que l'infestation demeure inférieure au seuil de nuisibilité puisqu'on attire proportionnellement moins de papillons provenant de situations éloignées. Un début de confirmation de cette hypothèse est fournie par l'expérimentation sur les « minidoses » effectuée par le Service de la Protection des Végétaux (BESSON, 1981). Cette expérimentation, menée sans comparaison avec les diffuseurs habituellement utilisés, en 4 points de l'Alsace (région à 2 générations) et de la région MidiPyrénées (région à 3 générations), a montré que, pour le $1^{\text {er }}$ vol en Alsace et le $2^{e}$ vol en Midi-Pyrénées, les classements du nombre de mâles piégés et des pourcentages d'attaque sont les mêmes. Dans le MidiPyrénées, où les autres vols n'ont pas montré de concordance avec l'infestation, les résultats sont proches des nôtres, obtenus en Aquitaine, région limitrophe du Midi-Pyrénées.

\section{CONCLUSIONS}

Malgré un dispositif expérimental différent, les essais rapportés ici confirment la constatation établie par des essais « en concurrence » à savoir (ROEHRICH et al., 1983), que le nombre de captures par piège sexuel diffère peu, même si on utilise des doses très différentes, variant de 1 à $1000 \mu \mathrm{g}$. Prolongée par des mesures de l'infestation aux alentours des pièges cette étude montre que plus la dose est faible, meilleure est la concordance entre les données du piégeage et la population. Il deviendrait donc possible de diminuer le nombre des cas où le dénombrement des papillons capturés ne permet pas de décider si un traitement est utile ou non. Une expérimentation complémentaire, en diverses situations, est cependant nécessaire avant de pouvoir définir de nouveaux seuils d'intervention, plus discriminants que ceux établis à l'aide de diffuseurs chargés à $1000 \mu \mathrm{g}$.

\section{Reçu le 21 février 1985. Accepté le 8 janvier 1986.}

\section{REMERCIEMENTS}

Nous tenons à remercier Mme Martine LeTtere (I.N.R.A., Laboratoire des Médiateurs chimiques) pour la synthèse de la phéromone nécessaire à cette expérimentation, ainsi que $\mathrm{M}$. DESCOINS, Directeur de ce laboratoire. Nous remercions également Mme BALLANDE, propriétaire de Château Baret et M. REALT, régisseur, qui nous ont permis d'expérimenter dans leur vignoble et facilité ainsi notre travail. 


\section{RÉFÉRENCES BIBLIOGRAPHIQUES}

Audemard H., 1979. Note sur l'utilisation du piégeage sexuel du carpocapse des pommes et des poires (Laspeyresia pomonella). A.C.T.A., note d'inf. L.I., $\mathrm{n}^{\circ} 28,23-30$.

Baker T. C., Roelofs W. L., 1981. Initiation and termination of oriental fruit moth male response to pheromone concentrations in the fields. Environ. Entomol., 10, 211-217.

Besson J., 1981. Compte rendu des essais effectués en 1981 par le Service de la Protection des Végétaux à l'aide de capsules minidosées en phéromones sexuelles. Rapport du Service de la Protection des Végétaux (non publié).

Maurin G., 1980. Piégeage sexuel des tordeuses de la grappe (action menée en Gironde en 1979). Déf. Vég., 34, 152-166.

Ramaswany S. B., Cardé R. T., 1983. Relationships between catch in pheromone baited traps and larval density of the spruce budworm Choristoneura fumiferana (Lepidoptera : Tortricidae). Can. Entomol., 115, 1437-1443.
Roehrich R., 1981. Lutte intégrée en viticulture ; IVe réunion plénière, travaux du sous-groupe «Tordeuse de la grappe». Boll. Zool. agr. Bacchic., $\mathrm{II}^{\mathrm{e}}$ série, 16, 7-34.

Roehrich R., Schmid A., 1979. Lutte intégrée en viticulture : tordeuse de la grappe, évaluation du risque, détermination des périodes d'intervention et recherches de méthodes de lutte biologique, 245 253. Proc. Int. Symp. IOBC/WPRS integrierten Pflanz. Land, Forstwirtsch., Wien, 8-12 okt. 1979, 648 p.

Roehrich R., Carles J. P., Dreuilhe A., Vonderheyden F., 1983. Captures de Lobesia botrana Den. \& Schiff. (Lepidoptera : Tortricidae) au piège sexuel en relation avec la dose de phéromone du diffuseur. Agronomie, 3, 925-929.

Valli G., 1977. Considerazioni sull' uso di moderni mezzi biotechnici in agricoltura. Le trappole a feromoni sessuali. Boll. Zool. agr. Bacchic., II' série, 14, 133-144. 